\title{
Novel norcantharidin-loaded liver targeting chitosan nanoparticles to enhance intestinal absorption
}

This article was published in the following Dove Press journal:

International Journal of Nanomedicine

2 April 2012

Number of times this article has been viewed

Yong-yan Bei'
Xiao-yan Chen'
Yang Liu'
Jing-yu Xu'
Wen-juan Wang'
Zong-lin Gu'
Kong-lang Xing'
Ai-jun Zhu'
Wei-liang Chen'
Lin-seng Shi'
Qin Wang'
Xue-nong Zhang'
Qiang Zhang'
'College of Pharmaceutical Science,
Soochow University, Suzhou,
'Department of Pharmaceutics, School
of Pharmaceutical Science, Peking
University, Beijing, People's Republic
of China

Correspondence: Xue-nong Zhang College of Pharmaceutical Science, Soochow University, DuShuHu High Education Zone, Suzhou 215123, Jiang Su Province, People's Republic of China Tel +865I266992087 Email zhangxuenong@163.com

\begin{abstract}
In this paper, two novel liver-targeting nanoparticles, norcantharidin-loaded chitosan nanoparticles (NCTD-CS-NPs) and norcantharidin-associated galactosylated chitosan nanoparticles (NCTD-GC-NPs), were prepared using ionic cross-linkage. The physical properties, particle size, encapsulation efficiency, and drug release characteristics of the nanoparticles were investigated in vitro. To investigate the intestinal absorption mechanisms of the two preparations, a series of experiments was carried out, including in situ circulation method, in vitro everted gut sacs, and Ussing chamber perfusion technique. The absorption rate constants (Ka) of NCTD at different segments were found to be duodenum $>$ jejunum $>$ ileum $>$ colon. The concentration had no distinctive effect on absorption kinetics, suggesting that drug absorption is not dosedependent. The transport of NCTD was found to be inhibited by P-glycoprotein (P-gp) inhibitor, indicating that NCTD might be the substrate of P-gp. The order of the absorption enhancer effects were as follows: low molecular weight chitosan $(\mathrm{CS}-8 \mathrm{kDa})>$ high molecular weight chitosan $(\mathrm{CS}-30 \mathrm{kDa})>$ Poloxamer $>$ sodium dodecyl sulfate $(\mathrm{SDS})>$ sodium deoxycholate (SDCh). The results indicate that the chitosan nanoparticles can improve intestinal absorption of NCTD.
\end{abstract}

Keywords: P-glycoprotein, absorption enhancers

\section{Introduction}

Norcantharidin (NCTD), a demethylation derivative of cantharidin obtained from the dried body of the Chinese blister beetle (mylabris), is used as a traditional medicine in China. ${ }^{1}$ Clinical studies have shown that NCTD is effective against the primary carcinoma of liver as an inhibitor of protein phosphatase 1 (PP1) and protein phosphatase 2A (PP2A). ${ }^{2}$ Recent studies have also indicated that NCTD administered orally or intravenously has potential applications in cancer chemotherapy. ${ }^{3-7}$ However, the clinical use of NCTD is limited because of its significant side effects, including irritation of the urinary organs. ${ }^{8}$ Moreover, NCTD usually has to be administered in high doses to strengthen its tumor inhibition effects due to its low intestinal permeability. The administration of high doses causes more intense irritation at the injection site.

Chitosan (CS), as a nontoxic and biodegradable polycationic polymer with low immunogenicity, has been investigated extensively as a formula carrier and delivery system for therapeutic molecules, such as gene, protein molecule, vaccine, and ammonium glycyrrhizinate..$^{9,10}$ Among the various mentions of chitosan derivatives in literature, ${ }^{11,12}$ one can differentiate specific reactions involving the free $-\mathrm{NH}_{2}$ group at the $\mathrm{C}-2$ position. Lactosaminated N-succinylchitosan was found to be a good drug carrier in the treatment of liver diseases, ${ }^{13,14}$ because the lactose base can 
bind with asialoglycoprotein receptors in the liver surface to achieve active liver targeting. To improve the therapeutic efficacy of NCTD and decrease its side effects, two novel norcantharidin-loaded liver targeting chitosan nanoparticles (NCTD-NPs), including norcantharidin-loaded chitosan nanoparticles (NCTD-CS-NPs) and norcantharidin-associated galactosylated chitosan nanoparticles (NCTD-GC-NPs), were successfully developed in our previous studies. ${ }^{15,16}$ By virtue of their unique uptake mechanisms and liver-specific targeting properties, NCTD nanoparticles have received much attention as a suitable alternative drug carrier system.

Although the physical properties and numerous oral dosage forms of NCTD have been studied, the understanding of NCTD absorption is very limited. Drug absorption is a key process governing in vivo bioavailability of drugs. It is affected by the physicochemical properties of the drug, dosage form, biological state of the gastrointestinal (GI) tract, and coadministered food components. ${ }^{17}$ Various physicochemical parameters, such as molecular size and shape, lipophilicity, hydrogen bonding capacity, and molecular surface properties, appear to play crucial roles in the absorption of drugs that cross the intestinal barrier through passive diffusion, either through the transcellular or paracellular pathway. ${ }^{18-21}$ In recent years, several studies have been devoted to the assessment of relationships between such parameters and drug absorption in various model systems. ${ }^{22-25}$

In our present study, the absorption mechanisms of two nanoparticles of NCTD, NCTD-CS-NPs and NCTDGC-NPs, were investigated in comparison with that of NCTD. The absorption characteristics of NCTD-NPs in the intestinal barrier were studied using in vivo transport experiments, in vitro everted sac experiments, and Ussing chamber perfusion technique.

\section{Materials and methods Materials}

Norcantharidin (lot number 20100515) with a purity of 99.21\% was purchased from Surui Medicine Chemical Co, Ltd (Suzhou, Jiangsu, China). Chitosan with a deacetylation of $90.9 \%$ and a molecular weight $(\mathrm{Mw})$ from 8 to $30 \mathrm{kDa}$ was supplied by Xingcheng Biochemical Co, Ltd (Nantong, China). Galactosylated chitosan (GC) was synthesized as previously described by Chung et al. ${ }^{26}$ Sodium tripolyphosphate (TPP) was obtained from the National Drug Group (Shanghai, China) and Pluronic F68 was purchased from Fluka Chemika (Buchs, Switzerland). Other chemicals and solvents were of analytical grade. Deionized twice-distilled water was used throughout the study.
Male Sprague-Dawley (SD) rats weighing from 220 to $270 \mathrm{~g}$ were obtained from the Laboratory Animals Center of the Medical College, Soochow University (Suzhou, China). All animal procedures were conducted in accordance with the guidelines for the care and use of animals of the National Institutes of Health.

\section{Samples preparation}

NCTD-NPs were prepared according to a procedure ${ }^{15,16}$ based on the ionic gelation of CS/GC with TPP anions, which was slightly modified. Briefly, $200 \mathrm{mg} \mathrm{CS} / \mathrm{GC}$ was dissolved in $100 \mathrm{~mL}$ acetic acid aqueous solution containing $0.4 \mathrm{mg} / \mathrm{mL}$ NTCD. TPP aqueous solution was then added to the chitosan solution drop by drop under magnetic stirring, resulting in cross-linkage. After reaction for 10 minutes, $125 \mathrm{mg}$ Pluronic F68 was dissolved in the solution as a stabilizer and $2 \%$ acetic acid aqueous solution concentration containing chitosan:TPP (50:20 V/V) was stirred at a constant rate of $500 \mathrm{rpm}$ for 2 hours.

In the method above, NCTD gelata was prepared using equivalent distilled water instead of TPP.

The perfusion solution was a Krebs-Ringer buffer solution, which contained $7.8 \mathrm{~g} \mathrm{NaCl}, 0.35 \mathrm{~g} \mathrm{KCl}, 1.37 \mathrm{~g}$ $\mathrm{NaHCO}_{3}, 0.02 \mathrm{~g} \mathrm{MgCl}_{2}, 0.22 \mathrm{~g} \mathrm{NaH}_{2} \mathrm{PO}_{4}$, and glucose in $1.48 \mathrm{~g} / 1000 \mathrm{~mL}$ purified water.

\section{Particle size analysis and morphological characteristics}

Particle size analysis of the NPs was performed by dynamic light-scattering measurement using the Zetasizer Nanoparticle Analyser (Malvern Instruments Ltd, Malvern, UK) with a range from 0.6 to $600 \mathrm{~nm}$. Morphological characteristics of the nanoparticles were studied through transmission electron microscopy (TEM; JEM-1230, JEOL, Japan). Colloid samples of the NPs were condensed using a Vivaflow 50 Ultrafilter (30,000 Da polysulfone resin filter membrane; Sartorius AG, Göttingen, Germany). A drop of nanoparticle suspension was placed onto a carbon film coated on a copper grid for TEM. Negative staining was performed using phosphotungstic acid $(0.05 \%, \mathrm{w} / \mathrm{w})$. Observation was performed at $80 \mathrm{kV} .^{27}$

\section{Evaluation of drug entrapment efficiency and loading capacity}

The concentration of NCTD in the nanoparticles was determined by a reversed-phase HPLC method. The HPLC system was composed of two pumps (LC-10AT VP, Shimadzu, Japan) and a UV-vis detector (SPD-M10A VP, Shimadzu) set at $210 \mathrm{~nm}$. The chromatographic column was a Luna 
C18 (4.6 × 250 mm; Hypersil ODS2, Elite, Dalian, China) maintained at $30^{\circ} \mathrm{C}$. The mobile phase was a mixture of acetonitrile: $\mathrm{H}_{2} \mathrm{O}=10: 90$ (adjusted to $\mathrm{pH} 3.1$ by adding phosphoric acid), and the flow rate was $0.8 \mathrm{~mL} / \mathrm{min}$.

An appropriate volume of NPs colloid was filtered through a $0.45 \mu \mathrm{m}$ filter to remove nonsoluble aggregate residues following the method in the previous report. ${ }^{28,29}$ The filtered colloid was then ultracentrifuged at 40,000 rpm for 45 minutes using the Optima MAX Centrifuge (Berkman Co, Ltd, USA). The supernatant was sampled and the NCTD concentration in the supernatant was determined by HPLC.

Encapsulation efficiency $(E E \%)$ and drug loading efficiency $(D L \%)$ were calculated using the following equations:

$$
\begin{gathered}
E E=\frac{T-F}{T} \times 100 \% \text { and } \\
D L=\frac{T-F}{W} \times 100 \%
\end{gathered}
$$

where $T$ is the total NCTD in the colloid, $F$ is the free NCTD in the supernatant, and $W$ is the weight of NPs.

\section{In vitro release studies}

Drug release from NCTD- NPs in vitro was measured using a dialysis method and compared with that of the NCTD solution. Briefly, $6 \mathrm{~mL}$ NCTD-NPs was placed into a dialysis tube (8000-14,000 MWCO) and sealed at both ends with clips. The dialysis tube loaded with nanoparticles was then placed into a beaker containing $30 \mathrm{~mL}$ deionized twice-distilled water and incubated with stirring for 3 hours at $37^{\circ} \mathrm{C} \pm 0.5^{\circ} \mathrm{C}$. At various time points, $0.5 \mathrm{ml}$ aliquots were withdrawn from the beaker and replaced with an equal volume of media. The NCTD concentrations were then measured by HPLC. The cumulative percentage of NCTD released was calculated as the amount of NCTD released from the solution or nanoparticles relative to the total amount of NCTD present in the formulation.

\section{In vivo transport studies}

An in vivo recirculation perfusion technique was used and performed as reported previously. ${ }^{30-32}$ This method was used to determine absorption characteristics at four different segments: ${ }^{33}$ duodenum, jejunum, ileum, and colon. Three different concentrations were studied: $140 \mu \mathrm{g} / \mathrm{mL}, 160 \mu \mathrm{g} / \mathrm{mL}$, and $180 \mu \mathrm{g} / \mathrm{mL}$. The perfusate was prepared by dispersing NCTD/NCTD-NPs with different drug concentrations in the Krebs-Ringer BH solution $(100 \mathrm{~mL})$ containing phenol red $(20 \mu \mathrm{g} / \mathrm{mL})$ for the purpose of correcting the perfusion volume. Next, $0.5 \mathrm{~mL}$ of the sample solution was used to detect the phenol red concentration by UV detection at $559 \mathrm{~nm}$. Another $0.5 \mathrm{~mL}$ was digested by $88 \%$ methanoic acid $(0.33 \mathrm{~mL})$ and then centrifuged for 10 minutes at $4000 \mathrm{rpm}$. Next, $100 \mu \mathrm{L}$ of the supernatant was separated and placed into another centrifuge tube, into which $200 \mu \mathrm{L}$ methanol was added. After vortexing for 3 minutes, the extraction was centrifuged for 3 minutes at 10,000 rpm. Afterward, $20 \mu \mathrm{L}$ of the supernatant was injected onto the HPLC for determination of NCTD using the same HPLC method described previously in "Evaluation of drug entrapment efficiency and loading capacity." The absorption percentage $\left(\mathrm{P}_{\mathrm{A}}\right)$, absorption rate constant $(\mathrm{Ka})$ and absorption half-life $\left(t_{1 / 2 \mathrm{a}}\right)$ for the NCTD solution/NCTD-NPs were calculated.

\section{In vitro transport studies}

Everted sacs of the rats were prepared using the method described previously, ${ }^{34}$ which was also used to determine the absorption characteristics at different segments and with different concentrations. All samples were treated using the method described in "In vivo transport studies."

\section{P-gP dependency of NCTD}

To examine the saturation potential of the P-gp function, the transport of NCTD and NCTD-NPs at the duodenum was investigated using the everted sacs method. The P-gp inhibitor, Cyclosporin A(CyA), a well-known P-gp substrate, was used as the positive control group at concentrations of 5,10 , and $20 \mu \mathrm{g} / \mathrm{mL}$.

\section{Effects of absorption enhancers}

The effects of improving absorption of NCTD and NCTD-NPs used as enhancers at the duodenum were investigated using the chamber method. Five enhancers, namely, low molecular weight chitosan (CS-8kDa), high molecular weight chitosan (CS$30 \mathrm{kDa}$ ), Poloxamer, sodium dodecyl sulfate (SDS), and Sodium deoxycholate (SDCh), were used with concentrations of $0.1 \%$, $0.5 \%$, and $1.0 \%$. Apparent permeability coefficients $\left(P_{\text {app }}\right)$ were calculated according to the equation below:

$$
P_{\text {app }}=\frac{d Q}{d t}\left(\frac{1}{A C_{0}}\right)
$$

where $d Q / d t$ is the permeability rate, A is the diffusion area, and $C_{0}$ is the initial donor solution concentration. 
Table I Physicochemical characteristics of NCTD nanoparticles $(\mathrm{n}=3)$

\begin{tabular}{lllcl}
\hline $\begin{array}{l}\text { NCTD } \\
\text { nanoparticles }\end{array}$ & $\begin{array}{l}\text { Mean particle size } \\
(\mathbf{n m})\end{array}$ & $\begin{array}{l}\text { Encapsulation efficiency } \\
(\%)\end{array}$ & $\begin{array}{l}\text { Drug loading efficiency } \\
(\%)\end{array}$ & $\begin{array}{l}\text { Yield } \\
\text { (\%) }\end{array}$ \\
\hline NCTD-CS-NPs & $131 \pm 11$ & $45.12 \pm 0.33$ & $7.3 \pm 0.13$ & $99.62 \pm 0.15$ \\
NCTD-GC-NPs & $119 \pm 9$ & $57.92 \pm 0.40$ & $10.38 \pm 0.06$ & $98.71 \pm 0.22$ \\
\hline
\end{tabular}

Abbreviations: NCTD-CS-NPs nanoparticles, norcantharidin-loaded chitosan nanoparticles; NCTD-GC-NPs, norcantharidin-associated galactosylated chitosan nanoparticles.

\section{Statistics}

Values are expressed as $\bar{x} \pm s$ (mean $\pm \mathrm{SD}$ ). Data were analyzed by one-way ANOVA with the post-hoc Tukey's test applied for paired comparisons (SPSS 16, SPSS Inc, Chicago, USA). Moreover, the criterion of statistical significance was taken as ${ }^{*} P<0.05,{ }^{* *} P<0.01$, or ${ }^{\sharp} P<0.05$, ${ }^{\#} P<0.01$.

\section{Results and discussion Physicochemical properties of NCTD-NPs}

The physicochemical characteristics of NCTD-NPs, including particle size and yield, drug loading, and encapsulation efficiency, are summarized in Table 1. The two livertargeting NCTD-loaded nanoparticles, NCTD-CS-NPs and NCTD-GC-NPs, had small particle sizes of about $100 \mathrm{~nm}$ and displayed an encapsulation efficiency of about $50 \%$, leading to the final NCTD loading values of about $10 \%$. The values of the NCTD-GC-NPs were higher than those of the NCTD-CS-NPs.

The majority of the fenestrate of the liver sinusoid was reported to be typically less than $200 \mathrm{~nm}$ in diameter. ${ }^{35}$ Permeability of the vascellum endothelium existing in the liver tumor was also enhanced greatly. ${ }^{36}$ Thus, the novel NCTD-CS-NPs could pass through the fenestrate sinusoid and accumulate in the tumor sites of the liver. The small particle size could reduce the adverse reaction of NCTD-NPs on normal hepatic cells and reduce passive liver-targeting. The two NCTD-NPs appeared spherical in shape, as shown in Figure 1.

\section{In vitro drug release of NCTD-NPs}

The data results of in vitro NCTD release characteristics are presented in Figure 2. The release curve conforms to the
A

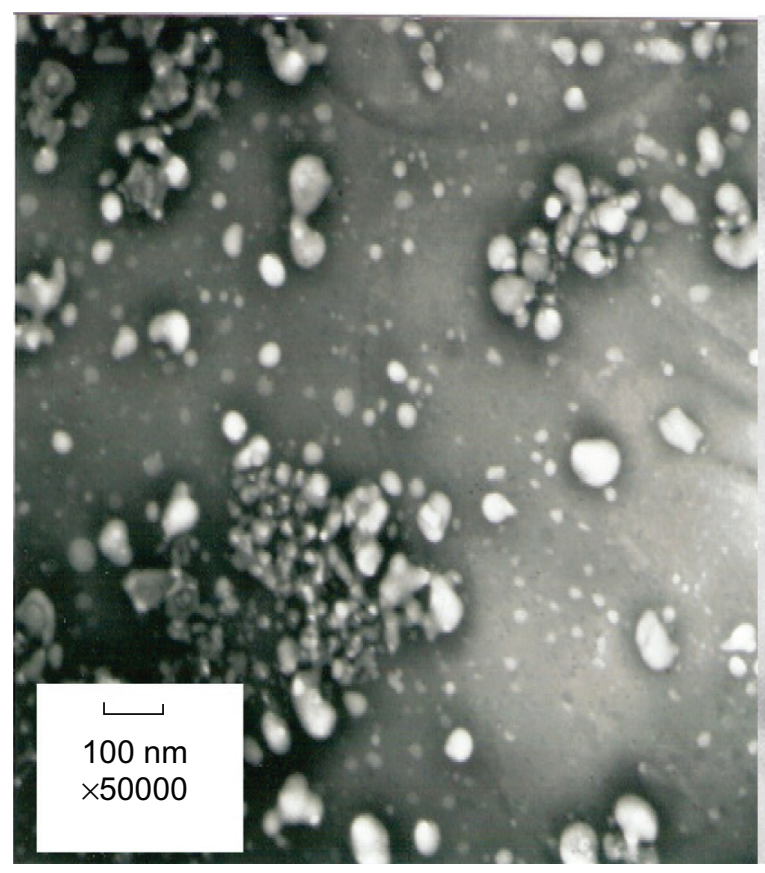

B

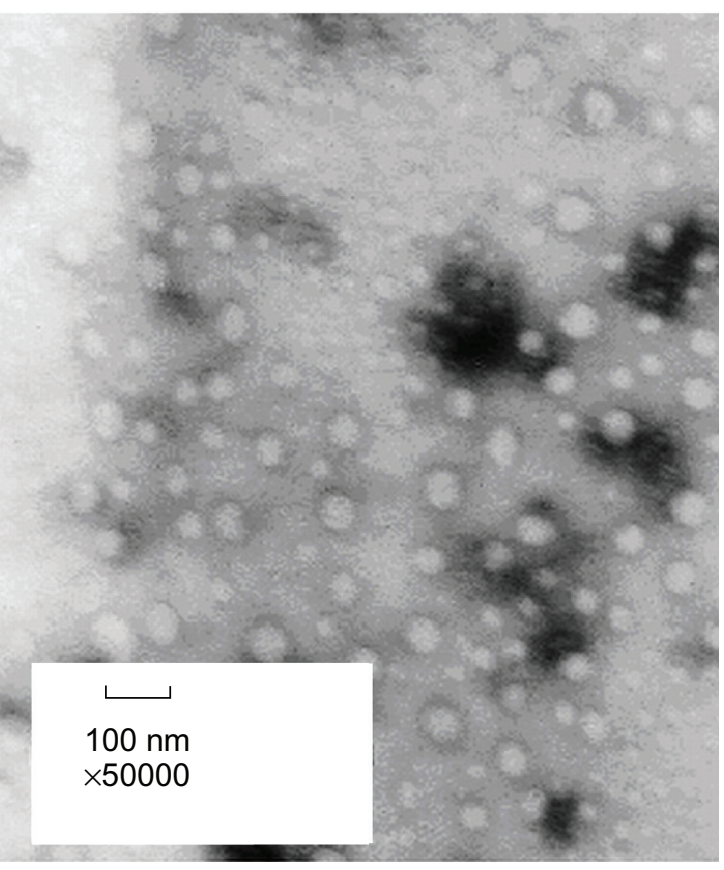

Figure I TEM photograph of NCTD-CS-NPs (A) and NCTD-GC-NPs (B).

Abbreviations: NCTD-CS-NPs nanoparticles, norcantharidin-loaded chitosan nanoparticles; NCTD-GC-NPs, norcantharidin-associated galactosylated chitosan nanoparticles; TEM, transmission electron microscope. 


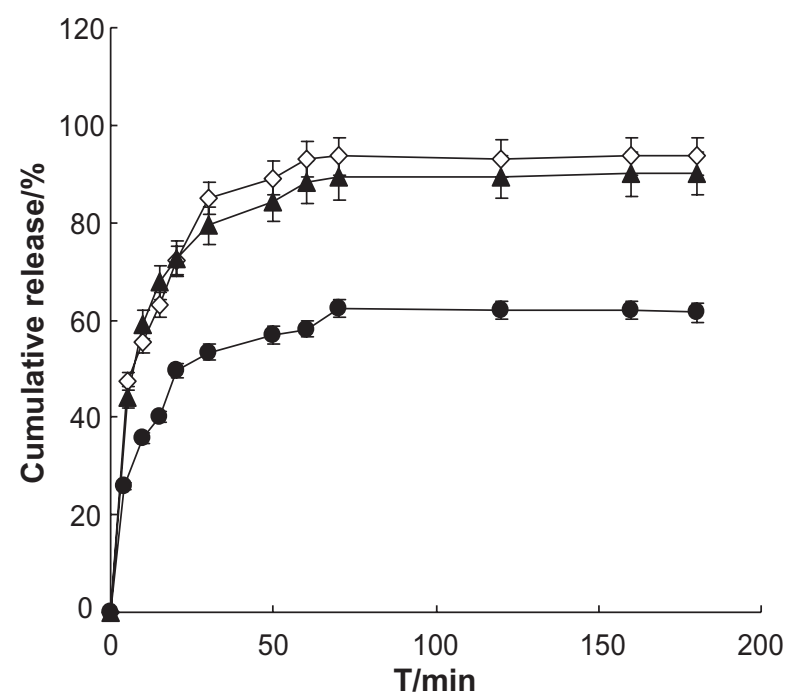

Figure 2 In vitro drug release of NCTD-CS-NPs $(\diamond)$ and NCTD-GC-NPs $(\boldsymbol{\Delta})$ compared with NCTD gelata $(\bullet)$.

Abbreviations: NCTD-CS-NPs nanoparticles, norcantharidin-loaded chitosan nanoparticles; NCTD-GC-NPs, norcantharidin-associated galactosylated chitosan nanoparticles.

Weibull model; the soluble equation is plotted using the data and a good fit is achieved. $\mathrm{T}_{\mathrm{d}}, \mathrm{T}_{50}$ and other parameters are calculated according to the soluble equation; the results are shown in Table 2. Analysis of the data shows that $\mathrm{T}_{\mathrm{d}}, \mathrm{T}_{50}$ of the two formulations are significantly different $(P<0.01)$ and the parameters of NCTD-NPs are significantly less than the gelata, which means that NCTD-NPs are more conducive for the release of the drugs than the gelata. ${ }^{37}$ In conclusion, the in vitro release of nanoparticles was found to follow the Weibull equation (data not shown) by data fitting $(r>0.9500)$. The drug release of NCTD-NPs was more complete than that of NCTD gelata. It was also argued that NCTD-NPs were more conducive to drug delivery than NCTD gelata. ${ }^{38}$

\section{Absorption properties of NCTD-NPs in rat intestines Absorption of NCTD-NPs in various intestinal segments of rats}

Regional differences in intestinal drug permeability have been attributed to the difference in epithelial surface area, pore radius of the paracellular pathway, mucus layer, and regional membrane fluidity. ${ }^{39}$ In this NCTD-NPs study, the absorption tests were performed on four different intestinal segments to test whether the intestinal absorption of NCTD-NPs exhibited site-dependent changes. The intestinal absorption kinetics of NCTD-NPs and NCTD solution at a dose of $160 \mu \mathrm{g} / \mathrm{mL}$ were investigated. The results are shown in Table 3, from which it could be concluded that NCTD absorption through the duodenum and the jejunum were much higher than those through the ileum and colon. The Ka of NCTD absorption was duodenum $>$ jejunum $>$ ileum $>$ colon. In addition, drug absorption is affected mainly by the physicochemical properties of drugs, drug metabolism in the gastrointestinal mucosa, lipophilicity, unionized fraction of drugs, molecular size, and drug solubility. ${ }^{40}$ Changing these absorption parameters along the gastrointestinal tract may contribute to the differences in site-specific absorption of melatonin. Regional differences in the functional expression of P-gp might be one of the possible factors for site-dependent absorption. Compared to the in vitro everted sac method, $\mathrm{Ka}$ of the in vivo method was much higher while $t_{1 / 2 a}$ was much lower. This is because during the in vitro method, the mesentery and blood vessels were cut off, which leads to less absorption. Among the three formulations, the order of Ka was NCTD-GC-NPs $>$ NCTD-CS-NPs $>$ NCTD solution. It has been suggested that chitosan nanoparticles could improve the intestinal absorption of NCTD because chitosan can trigger the opening of tight junctions ${ }^{41}$ In addition, there are plenty of Peyer's patches and $\mathrm{M}$ cells in rat intestines, and absorption via the lymphatic tissues is an important route for drugs with low oral bioavailability ${ }^{42-44}$ Nanoparticles, as compositions of intestine mucous membrane, have an important effect on the enhancement of absorption via the lymphatic route..$^{45}$

\section{Absorption of NCTD-NPs in various concentrations in rats}

As shown in Figure 3, the intestinal absorption percentages of NCTD at higher concentration were greater than those at lower concentrations in vivo or in vitro. However, the

Table 2 Soluble equation and parameters $(n=5)$

\begin{tabular}{|c|c|c|c|c|}
\hline Group & Weibull equation & $\mathbf{r}$ & $\mathbf{T}_{d} / \mathrm{min}$ & $\mathrm{T}_{50} / \mathrm{min}$ \\
\hline NCTD-CS-NPs & $\ln \ln [I / I-F(t)]=0.532 \ln t-1.3334$ & 0.97379 & $12.25 \pm 0.3$ & $6.15 \pm 0.2$ \\
\hline NCTD-GC-NPs & $\ln \ln [\mathrm{I} / \mathrm{I}-\mathrm{F}(\mathrm{t})]=0.3775 \ln \mathrm{t}-0.9438$ & 0.95917 & $12.17 \pm 0.5$ & $4.6 \mathrm{I} \pm 0.3$ \\
\hline NCTD gelata & $\ln \ln [1 / I-F(t)]=0.370 \ln t-1.6412$ & 0.97528 & $84.33 \pm 0.7$ & $31.35 \pm 0.6$ \\
\hline
\end{tabular}

Abbreviations: NCTD-CS-NPs nanoparticles, norcantharidin-loaded chitosan nanoparticles; NCTD-GC-NPs, norcantharidin-associated galactosylated chitosan nanoparticles. 
Table 3 Absorption parameters at various segments in rat intestines $(n=3)$

\begin{tabular}{|c|c|c|c|c|c|c|c|}
\hline \multirow[t]{2}{*}{ Segment } & \multirow[t]{2}{*}{ Groups } & \multicolumn{2}{|l|}{$P_{A} / \%$} & \multicolumn{2}{|l|}{$K_{a} / h^{-1} \times 100$} & \multicolumn{2}{|l|}{$t_{1 / 2 a} / \mathrm{h}$} \\
\hline & & In vivo (6 hours) & In vitro (2 hours) & In vivo & In vitro & In vivo & In vitro \\
\hline \multirow[t]{3}{*}{ Duodenum } & NCTD solution & $15.69 \pm 1.12$ & $4.76 \pm 0.22$ & $3.06 \pm 0.33$ & $2.81 \pm 0.21$ & $22.67 \pm 2.44$ & $24.70 \pm 1.85$ \\
\hline & NCTD-CS-NPs & $29.16 \pm 1.87$ & $13.21 \pm 1.13$ & $6.89 \pm 0.21$ & $5.98 \pm 0.37$ & $10.06 \pm 0.31$ & $11.59 \pm 0.72$ \\
\hline & NCTD-GC-NPs & $35.79 \pm 0.34$ & $16.48 \pm 075$ & $6.97 \pm 0.42$ & $9.05 \pm 0.51$ & $9.95 \pm 0.15$ & $7.66 \pm 0.37$ \\
\hline \multirow[t]{3}{*}{ Jejunum } & NCTD solution & $12.49 \pm 0.89$ & $4.70 \pm 0.15$ & $2.78 \pm 0.26$ & $2.60 \pm 0.13$ & $24.93 \pm 2.33$ & $26.61 \pm 1.33$ \\
\hline & NCTD-CS-NPs & $24.34 \pm 1.01$ & $10.88 \pm 0.34$ & $5.92 \pm 0.35$ & $4.24 \pm 0.23$ & $11.71 \pm 0.69$ & $16.36 \pm 0.89$ \\
\hline & NCTD-GC-NPs & $29.43 \pm 0.15$ & $16.65 \pm 1.21$ & $6.16 \pm 0.52$ & $9.10 \pm 0.36$ & $|I .25 \pm 0.5|$ & $7.62 \pm 0.53$ \\
\hline \multirow[t]{3}{*}{ Ileum } & NCTD solution & $7.73 \pm 0.25$ & $2.76 \pm 0.12$ & $1.51 \pm 0.14$ & $1.54 \pm 0.17$ & $45.89 \pm 4.25$ & $44.90 \pm 4.96$ \\
\hline & NCTD-CS-NPs & $13.98 \pm 0.97$ & $8.20 \pm 0.27$ & $3.53 \pm 0.17$ & $2.76 \pm 0.07$ & $19.63 \pm 0.95$ & $25.13 \pm 0.64$ \\
\hline & NCTD-GC-NPs & $17.67 \pm 0.27$ & $8.32 \pm 0.13$ & $4.09 \pm 0.25$ & $4.08 \pm 0.11$ & $16.94 \pm 0.77$ & $17.00 \pm 0.75$ \\
\hline \multirow[t]{3}{*}{ Colon } & NCTD solution & $2.20 \pm 0.33$ & $1.18 \pm 0.08$ & $0.44 \pm 0.01$ & $0.71 \pm 0.02$ & $157.5 \pm 3.58$ & $97.54 \pm 2.75$ \\
\hline & NCTD-CS-NPs & $3.54 \pm 1.23$ & $2.28 \pm 0.06$ & $0.95 \pm 0.01$ & $0.80 \pm 0.01$ & $72.95 \pm 0.78$ & $86.59 \pm 1.08$ \\
\hline & NCTD-GC-NPs & $9.59 \pm 0.23$ & $3.61 \pm 0.03$ & $1.36 \pm 0.23$ & $1.74 \pm 0.15$ & $50.90 \pm 1.23$ & $39.73 \pm 2.41$ \\
\hline
\end{tabular}

Abbreviations: NCTD-CS-NPs nanoparticles, norcantharidin-loaded chitosan nanoparticles; NCTD-GC-NPs, norcantharidin-associated galactosylated chitosan nanoparticles.

different concentrations did not induce a difference in the transport of NCTD $(P>0.05)$. It has been suggested that the concentration has no distinctive effect on absorption kinetics. A comparison of absorption kinetics among different concentrations demonstrated a non-concentration-dependent change. Results indicated that the intestinal absorption of NCTD-NPs is via passive transfer by diffusion across the intestinal membranes. ${ }^{46}$

\section{P-gP dependency of NCTD}

P-glycoprotein efflux pumps are located predominantly in the apical membranes of the epithelia (eg, small intestine, colon) and significantly limit oral absorption of a number of drugs. ${ }^{47}$

The transport of NCTD across everted gut sacs in solutions with added 5, 10, and $20 \mu \mathrm{g} / \mathrm{mL}$ CyA is shown in Figure 4. Results showed that the NCTD cumulative percentage in the duodenum at 120 minutes was significantly enhanced by CyA $(P<0.05)$. As the CyA concentration increased, the cumulative concentration of NCTD gradually increased. When the concentration of CyA was $20 \mu \mathrm{g} / \mathrm{mL}$, the accumulative concentration of NCTD at $120 \mathrm{~min}$ was more than $200 \%$ of the control (in the absence of CyA).
A

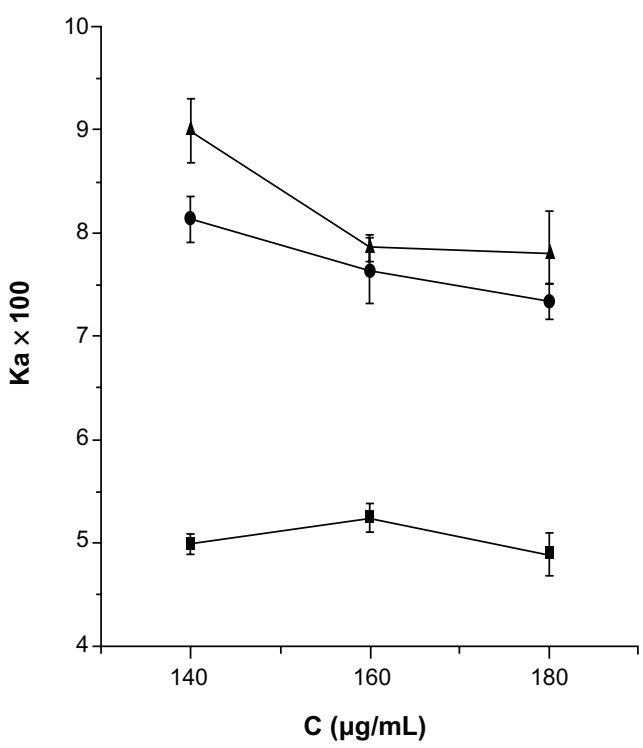

B

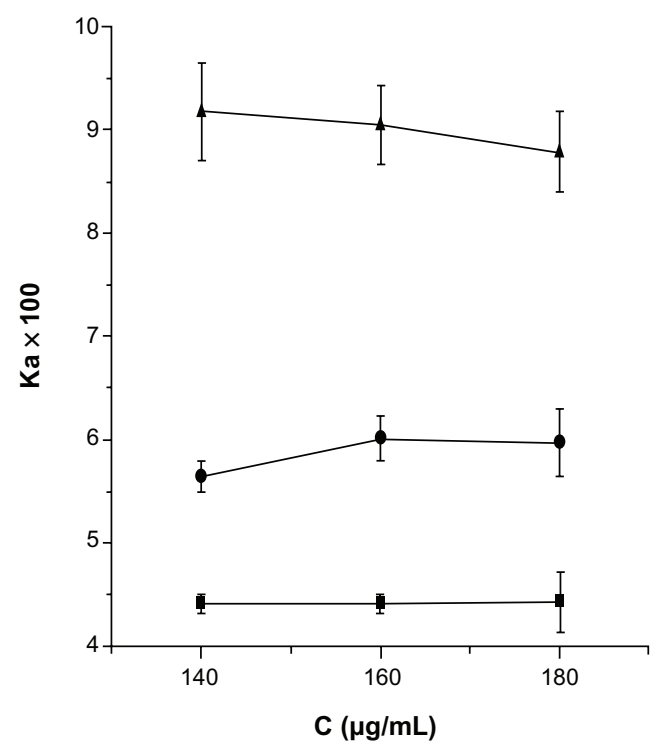

Figure 3 Intestinal absorption of NCTD-CS-NPs $(\times)$ and NCTD-GC-NPs $(\diamond)$ compared with that of NCTD solution $(\mathbf{\Delta})$ at different concentrations in vivo $(\mathbf{A})$ and in vitro $(\mathbf{B})$. Abbreviations: NCTD-CS-NPs nanoparticles, norcantharidin-loaded chitosan nanoparticles; NCTD-GC-NPs, norcantharidin-associated galactosylated chitosan nanoparticles. 


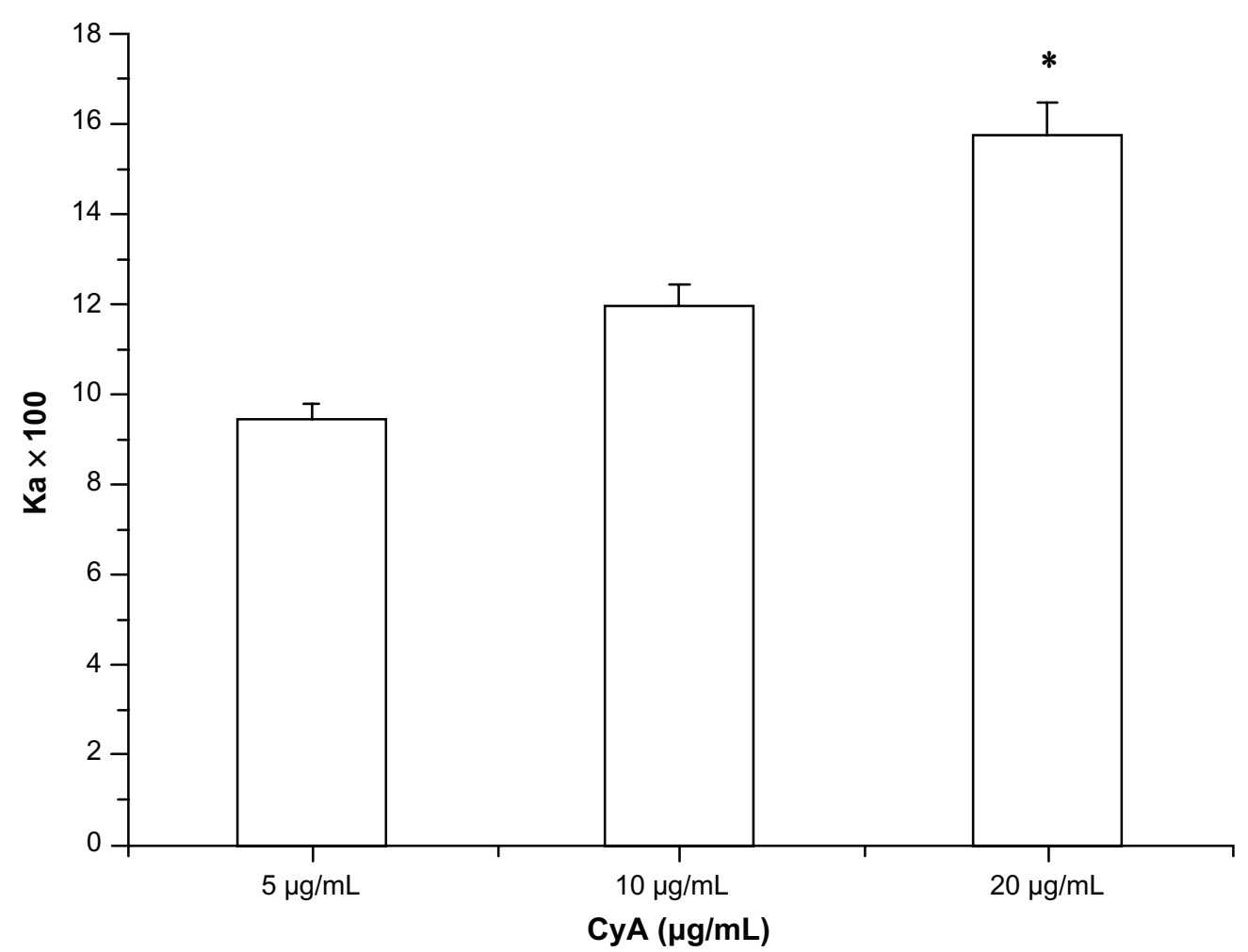

Figure 4 Intestinal absorption in vitro of NCTD with different concentrations of CyA.

Note: $* P<0.05$ versus $5 \mu \mathrm{g} / \mathrm{mL}$ group.

Abbreviations: NCTD-CS-NPs nanoparticles, norcantharidin-loaded chitosan nanoparticles; NCTD-GC-NPs, norcantharidin-associated galactosylated chitosan nanoparticles.

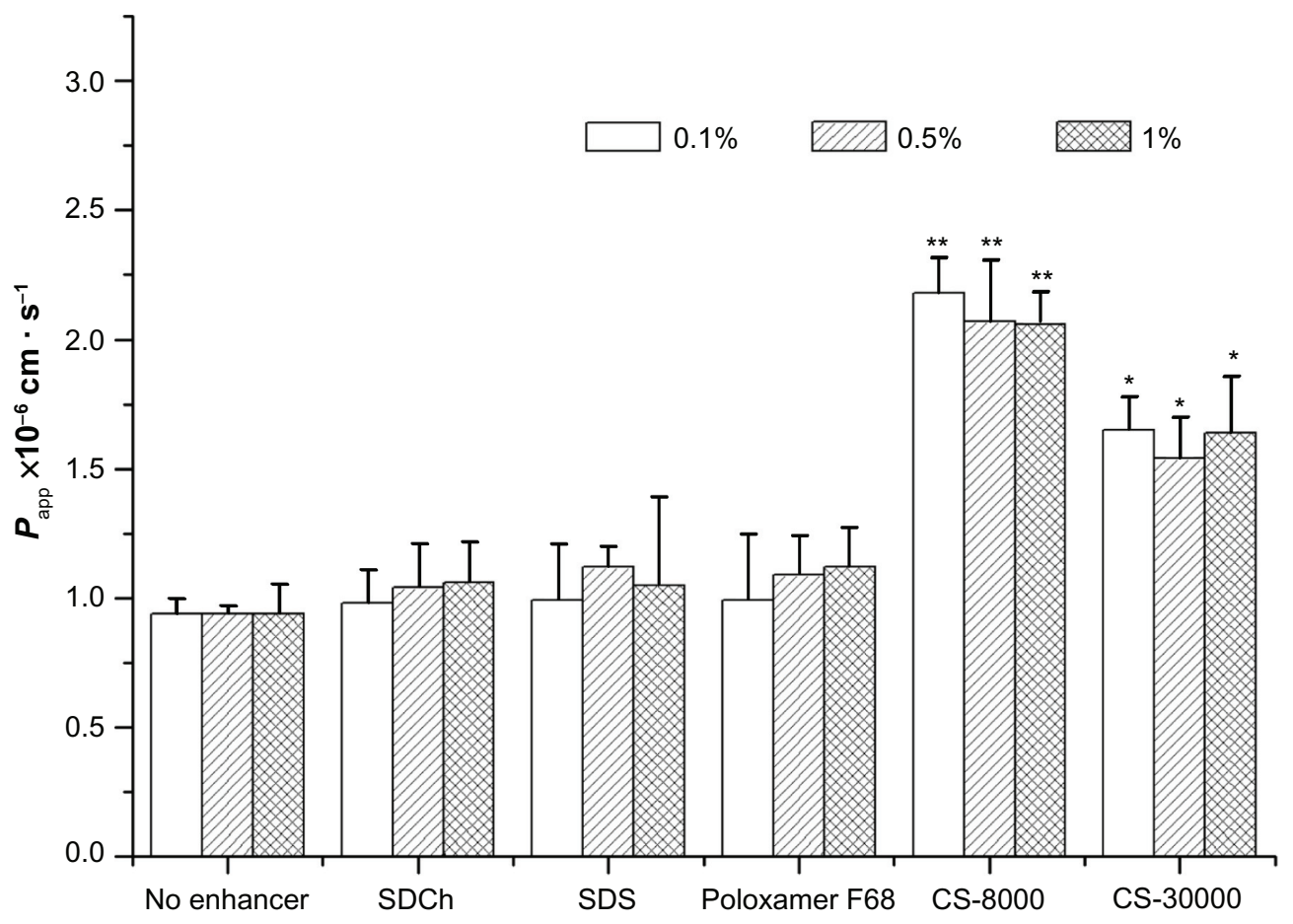

Figure 5 Effects of absorption enhancers on the apparent permeability coefficients $\left(P_{\text {app }}\right)$ for different concentrations.

Notes: $* P<0.05$ versus no enhancer group; $* * P<0.01$ versus no enhancer group.

Abbreviations: CS, chitosan; $P_{\text {app' }}$, permeability coefficients; SDCh, sodium deoxycholate; SDS, sodium dodecyl sulfate. 


\section{Effects of absorption enhancers}

The results are shown in Figure 5. Compared with the NCTD solution, absorption enhancers can enhance the absorption rate of the drug. The order of cross-mucosal permeability is CS-8$\mathrm{KDa}>\mathrm{CS}-30 \mathrm{KDa}>$ Poloxamer $>$ SDS $>$ SDCh. Among them, $0.1 \%$ CS-8KDa showed the most significant absorption promotion effect. With different concentrations of absorption enhancers to promote absorption between the different degrees, SDCh and Poloxamer absorption increased as the concentration was increased. SDS as an anionic surfactant promotes the role of low concentration; upon reaching a certain concentration, its effect decreased. The effect of CS-8KDa on absorption decreased as the concentration was increased. Thus it is proven that drugs containing low molecular weight chitosan as a carrier can help to promote intestinal absorption. The reason may be as follows: on the one hand, chitosan has a biological adhesive function, which can extend drug residence time in the intestines; on the other hand, because of its positively charged nature, it can connect with a variety of negatively charged protein bindings in the intestinal mucosa effectively, thereby inhibiting the physiological functions of certain proteins. ${ }^{48}$

\section{Conclusion}

Three intestinal absorption models in vivo and in vitro were used. They are simple, easy, and effective for drug absorption studies. The intestinal absorption mechanisms of NCTD solution and NCTD-NPs were investigated. Results of the study showed that NCTD-NPs significantly enhance drug absorption compared with the NCTD solution. Intestinal absorption kinetics provides methods for a more profound study of the process in vivo and in the development of new oral drugs.

\section{Acknowledgments}

This work was supported by the National Basic Research Program of China (973 Program No 2007CB935800), National Key Program of New Drug Innovation (No 2009zx09310-001), Technology Support Program of Jiangsu Province (BE2011670) and a project founded by the Priority Academic Program Development of Jiangsu Higher Education Institutions.

\section{Disclosure}

The authors report no conflicts of interest in this work.

\section{References}

1. Wang GS. Medical uses of mylabris in ancient China and recent studies. J. Ethnopharmacol. 1989;26(2):147-162.

2. Wang GS. Hydrolyze of norcantharidin and subtracting methyla alleviate stimulation of renal system. Chin Pharm Bull. 1983;18(7):18-20.
3. Yi SN, Wass J, Vincent P, Iland H. Inhibitory effect of norcantharidin on K562 human myeloid leukemia cells in vitro. Leuk Res. 1991;15(10):883-886.

4. Efferth T, Li PC, Konkimalla VS, Kaina B. From traditional Chinese medicine to rational cancer therapy. Trends Mol Med. 2007;13(8):353-361.

5. Shan HB, Cai YC, Liu Y, Zeng WN, et al. Cytotoxicity of cantharidin analogues targeting protein phosphatase 2A. Anticancer Drugs. 2006;17(8):905-911.

6. Efferth T, Rauh R, Kahl S, et al. Molecular modes of action of cantharidin in tumor cells. Biochem Pharmacol. 2005;69(5):811-818.

7. Kok SH, Hong CY, Kuo MY, et al. Comparisons of norcantharidin cytotoxic effects on oral cancer cells and normal buccal keratinocytes. Oral Oncol. 2003;39(1):19-26.

8. Liu XH, Blazsek I, Comisso M, et al. Effects of norcantharidin, a protein phosphatase type-2A inhibitor, on the growth of normal and malignant haemopoietic cells. Eur J Cancer. 1995;31A(6):953-963.

9. Chewa JL, Wolfowicz CB, Mao HQ, Leong KW, Chua KY. Chitosan nanoparticles containing plasmid DNA encoding house dust mite allergen, Der 1 for oral vaccination in mice. Vaccine. 2003;21(21-22): 2720-2729.

10. Wu Y, Yang WL, Wang CC, Hu JH, Fu SK. Chitosan nanoparticles as a novel delivery system for ammonium glycyrrhizinate. Int J Pharm. 2005;295(1-2):235-245.

11. Lixin W, Haibing H, Xing T, Ruiying S, Dawei C. A less irritant norcantharidin lipid microspheres: Formulation and drug distribution. Int J Pharm. 2006;323(1-2):161-167.

12. Bala I, Hariharan S, Kumar MN. PLGA nanoparticles in drug delivery: the state of the art. Cri Rev Ther Drug Carrier Sys. 2004;21(5): 387-422.

13. Kato Y, Onishi H, Machida Y. Lactosaminated and intact N-succinylchitosans as drug carriers in liver metastasis. Int J Pharm. 2001; 226(1-2):93-106.

14. Kato Y, Onishi H, Machida Y. Biological characteristic of lactosaminated $\mathrm{N}$-succinyl-chitosan as a liver-specific drug carrier in mice. J Control Release. 2001;70(3):295-307.

15. Zhang W, Liu Y, Chen XY, et al. Two modelling data analytical methods applied to optimise the preparation of norcantharidin chitosan nanoparticles. J Exp Nanosci. 2010;5(3):271-284.

16. Wang Q, Zhang L, Hu W, et al. Norcantharidin-associated galactosylated chitosan nanoparticles for dual hepatocyte-targeted delivery. Nanomedicine. 2010;6(2):371-S381.

17. Wagner D, Spahn LH, Hanafy A, Koggel A, Langguth P. Intestinal drug efflux: formulation and food effects. Adv Drug Deliv Rev. 2001; 50(Suppl 1):S13-S31.

18. Karls MS, Rush BD, Wilkinson KF, Vidmar TJ, Burton PS, Ruwart MJ. Desolvation energy: a major determinant of absorption, but not clearance, of peptides in rats. Pharm Res. 1991;8(12):1477-1481.

19. Conradi RA, Hilgers AR, Ho NF, Maggiora LL. The influence of peptide structure on transport across Caco-2 cell. II. Peptide bond modification which results in improved permeability. Pharm Res. 1992;9(3):435-439.

20. Stewart BH, Chan OH, Lu RH, et al. Comparison of intestinal permeabilities determined in multiple in vitro and in situ models: relationship to absorption in humans. Pharm Res. 1995;12(5):693-699.

21. Palm K, Luthman K, Ungell AL, Strandlund G, Artursson P. Correlation of drug absorption with molecular surface properties. J Pharm Sci. 1996;85(1):32-39.

22. Barrington R, Williamson G, Bennett RN, Davis BD, Brodbelt JS, Kroon PA. Absorption, Conjugation and Efflux of the Flavonoids, Kaempferol and Galangin, Using the Intestinal CACO-2/TC7 Cell Model. J Funct Foods. 2009;1(1):74-87.

23. Hillgren KM, Kato A, Borchardt RT. In vitro systems for studying intestinal drug absorption. Med Res Rev. 1995;15(2):83-109.

24. Rubas W, Cromwell MEM, Shahrokh Z, et al. Flux measurements across Caco-2 monolayers may predict transport in human large intestinal tissue. J Pharm Sci. 1996;85(2):165-169. 
25. Ungell AL. In vitro absorption studies and their relevance to absorption from the GI tract. Drug Dev Ind Pharm. 1997;23:879-892.

26. Chung TW, Yang J, Akaike T, et al. Preparation of alginate/ galactosylated chitosan scaffold for hepatocyte attachment. Biomaterials. 2002;23(14):2827-2834.

27. Zhang XN, Tang LH, Yan XY, Zhang Q. Albendazole polybutycyanocrylate nanoparticles-Preparation, pharmaceutical properties and tissue distribution in rats. Lett Drug Des Discov. 2006;3:275-279.

28. Banerjee T, Mitra S, Singh AK, Sharma RK, Maitra A. Preparation, characterization and biodistribution of ultrafine chitosan nanoparticles. Int J Pharm. 2002;243(1-2):93-105.

29. Lu E, Franzblau S, Onyuksel H, Popescu C. Preparation of aminoglycoside-loaded chitosan nanoparticles using dextran sulphate as a counterion. J Microencapsul. 2009;26(4):346-354.

30. Rubinstein A, Pathak YV, Kleinstern J, Reches A, Benita S. In vitro release and intestinal absorption of physostigmine salicylate from submicron emulsions. J Pharm Sci. 1991;80(7):643-647.

31. Nornoo AO, Zheng H, Lopes LB, Johnson-Restrepo B, Kannan K, Reed R. Oral microemulsions of paclitaxel: in situ and pharmacokinetic studies. Eur J Pharm Biopharm. 2009;71(2):310-317.

32. Ling W, Rui LC, Hua JX. In situ intestinal absorption behaviors of tanshinone IIA from its inclusion complex with hydroxypropylB-cyclodextrin. Biol Pharm Bull. 2007;30:1918-1922.

33. Michel C, Aprahamian M, Defontaine L, Couvreur P, Damgé C. The effect of site of administration in the gastrointestinal tract on the absorption of insulin from nanocapsules in diabetic rats. J Pharm Pharmacol. 1991;43(1):1-5.

34. Sasaki I, Tanaka K, Fujita T, Murakami M, Yamamoto A, Muranishi S. Intestinal absorption of azetirelin, a new thyrotropin-releasing hormone (TRH) analogue 11: in situ and in vitro absorption characteristics of azetirelin from the rat intestine. Biol Pharm Bull. 1995;18(7): 976-979.

35. Liang HF, Yang TF, Huang CT, Chen MC, Sung HW. Preparation of nanoparticles composed of poly( $\gamma$-glutamic acid)-poly(lactide) block copolymers and evaluation of their uptake by HepG2 cells. $J$ Control Release. 2005;105(3):213-225.

36. Wu J, Liu L, Yen R, et al. Polycationic liposome-mediated extracellular superoxide dismutase gene delivery prevents acute liver injury in mice. Hepatology. 2003;38(1):240-241.
37. Yang HY, Zhang MG. Targeting Drug Delivery System. Journal of China-Japan Friendship Hospital. 2001;15(5):292-294.

38. Li QZ. Using Weibull function to seek dissolution parameters. Chinese Journal of Hospital Pharmacy. 1991;11(1):30-31.

39. Masaoka Y, Tanaka Y, Kataoka M, Sakuma S, Yamashita S. Site of drug absorption after oral administration: assessment of membrane permeability and luminal concentration of drugs in each segment of gastrointestinal tract. Eur J Pharm Sci. 2006;29(3-4):240-250.

40. De Boer AG, editor. Drug Absorption Enhancement: Concepts, Possibilities, Limitations and Trends. Chur, Switzerland: Harwood Academic Publishers GmbH; 1994:367-389.

41. Cano-Cebrián MJ, Zornoza T, Granero L, Polache A. Intestinal absorption enhancement via the paracellular route by fatty acids, chitosans and others: a target for drug delivery. Curr Drug Deliv. 2005;2(1):9-22.

42. Hauss DJ, Fogal SE, Ficorilli JV, et al. Lipid-based delivery systems for improving the bioavailability and lymphatic transport of a poorly water-soluble LTB4 inhibitor. J Pharm Sci. 1998;87(2):164-169.

43. Holm R, Müllertz A, Pedersen GP, Kristensen HG. Comparison of the lymphatic transport of halofantrine administered in disperse systems containing three different unsaturated fatty acids. Pharm Res. 2001;18(9):1299-1304.

44. O'Driscoll CM. Lipid-based formulations for intestinal lymphatic delivery. Eur J Pharm Sci. 2002;15(5):405-415.

45. Khoo SM, Shackleford DM, Porter CJ, Edwards GA, Charman WN. Intestinal lymphatic transport of halofantrine occurs after oral administration of a unit-dose lipid-based formulation to fasted dogs. Pharm Res. 2003;20(9):1460-1465.

46. Wan HJ, Tang X, Yang DL. Absorption kinetics of ofloxacin in rats in situ. Chinese Journal of Hospital Pharmacy. 2005;25(7):597-599.

47. Ji HY, Li ZZ, Sun JR. Absorption mechanisms of protein and peptide in oral administration. Shandong Pharm Ind. 2003;22:31-32.

48. Collnot EM, Baldes C, Wempe MF, et al. Mechanism of inhibition of P-glycoprotein mediated efflux by vitamin ETPGS: influence on ATPase activity and membrane fluidity. Mol Pharm. 2007;4(3):465-474.
International Journal of Nanomedicine

\section{Publish your work in this journal}

The International Journal of Nanomedicine is an international, peerreviewed journal focusing on the application of nanotechnology in diagnostics, therapeutics, and drug delivery systems throughout the biomedical field. This journal is indexed on PubMed Central,

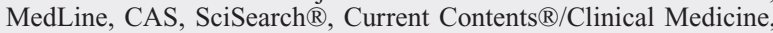

\section{Dovepress}

Journal Citation Reports/Science Edition, EMBase, Scopus and the Elsevier Bibliographic databases. The manuscript management system is completely online and includes a very quick and fair peer-review system, which is all easy to use. Visit http://www.dovepress.com/ testimonials.php to read real quotes from published authors. 